\title{
Oleína de Palma Estabilizada con Antioxidante Natural de Romero en un Proceso Discontinuo de Fritura
}

\author{
Andrea Zapata $^{(1) \star}$, Luz S. Vanegas ${ }^{(1)}$, Benjamin A. Rojano ${ }^{(2)}$ \\ (1) TECNAS SA, Innovación y desarrollo, Carrera 50G \# 12 sur 29, Itagüí, Colombia. \\ (e-mail: azapata@tecnas.com.co; gerenciainnovacion@tecnas.com.co) \\ (2) Universidad Nacional de Colombia, Sede Medellín. Facultad de Ciencias, Laboratorio de Ciencia de \\ Alimentos. Calle 59ª \# 63-20 Medellín, Colombia. (e-mail: brojano@unal.edu.co)
}

${ }^{*}$ Autor a quien debe ser dirigida la correspondencia.

Recibido Jul. 23, 2013; Aceptado Sep. 4, 2013; Versión final recibida Oct. 21, 2013

\section{Resumen}

En este estudio se evalúo el contenido de fenoles totales y la actividad antioxidante por diferentes metodologías como: DPPH, FRAP y ORAC de un producto comercial Noxy-Nat $\AA^{\circledR}$ reportado como antioxidante natural de romero. El Noxy-Nat a 1000 ppm inhibió en un $59 \%$ el deterioro oxidativo del aceite de palma, sometido a un proceso discontinuo de fritura de pollo a $180^{\circ} \mathrm{C}$. El proceso oxidativo fue monitoreado usando el valor de peróxido (VP) y los compuestos polares totales (CPT) como indicadores de la peroxidación lipídica y de la vida útil del aceite. El Noxy-Nat inhibe el deterioro oxidativo del aceite de palma debido a la capacidad para atrapar radicales peróxilos (ROO•) expresada como valores ORAC.

\section{Palm Olein Stabilized with Natural Rosemary Antioxidant in a Discontinuous Frying Process}

\begin{abstract}
In this study the total phenolic content and antioxidant activity by different methodologies such as DPPH, FRAP and ORAC for a commercial product Noxy-Nat@ reported as natural antioxidant rosemary were evaluated. Noxy-Nat to $1000 \mathrm{ppm}$ inhibited the oxidative deterioration of palm oil by $59 \%$, submitted to a batch of chicken frying process at $180^{\circ} \mathrm{C}$. The oxidative process was evaluated using the peroxide value (PV) and the total polar compounds (TPC) as indicators of lipid peroxidation and of the shelf life of the olein. Noxy-Nat inhibit oxidative deterioration of palm oil due to the ability to trap peroxyl radicals (ROO•) expressed as ORAC values.
\end{abstract}




\section{INTRODUCCION}

Freír es un método de cocción antiguo, que en los últimos cincuenta años ha ganado la atención de la comunidad científica. La fritura es un proceso integral donde se debe resaltar la importancia de cada una de las etapas y el tipo de ingredientes usados; es decir, la naturaleza del aceite y el alimento, tipo de freidora, la temperatura y los tiempos de exposición, la reposición del aceite y el uso de antioxidantes, entre otros (Bou et al., 2012, Wu et al., 2013, Alvis et al., 2008a, Juárez et al., 2005).

Sin embargo, los aceites usados en los procesos de fritura están sometidos a elevadas temperaturas durante periodos de tiempo prolongados, de tal manera que el oxígeno disuelto en el aceite y el agua de los alimentos, provocan una serie de reacciones oxidativas que producen diferentes tipos de compuestos como: carbonilos volátiles, hidroxiácidos, cetoácidos y epoxiácidos, que finalmente les confieren aromas y sabores desagradables a los aceites y al alimento (Giua et al., 2013, Alvis et al., 2008b). En la fritura el alimento en contacto con el aceite libera altas cantidades de agua que catalizan la hidrolisis de los acilgliceroles generando ácidos grasos libres insaturados $(\mathrm{RH})$, que a bajas concentraciones de oxígeno inician el proceso de peroxidación lipídica, produciendo inicialmente compuestos primarios del proceso como los dienos conjugados y los hidroperóxidos $(\mathrm{ROOH})$. Durante el proceso de oxidación, los hidroperóxidos generados son indicadores intermedios del deterioro oxidativo, los cuales pueden ser monitoreados y expresados como valor de peróxidos (VP). Los hidroperóxidos se descomponen en otras especies más oxidadas como aldehídos, cetonas y ácidos orgánicos, que mediante reacciones sucesivas forman compuestos poliméricos altamente oxidados. La concentración de todas estas especies se puede medir en el tiempo y se denominan Compuestos Polares Totales (CPT), los cuales son indicadores del deterioro total del aceite (Chen et al., 2013), ver figura 1.

De otro lado, el aceite para ser empleado en la fritura debe tener características químicas que contribuyan a su vida útil; por ejemplo, la relación inicial entre el contenido de ácidos grasos saturados e insaturados, la cual determina la estabilidad del producto durante el proceso de fritura. En diferentes estudios se proponen aceites con alto contenido de ácidos grasos mono insaturados especialmente ricos en ácido oleico como el aceite y las oleínas de palma, aceite de oliva y girasol (Cassal et al., 2010, Aladedunye et al., 2013). Por esta razón, el aceite y la oleína de palma incursionaron en el mercado mundial de la fritura a finales del siglo XX y se han mantenido debido a sus ventajas técnico-económicas comparados con otros aceites y grasas. Se estima que millones de toneladas de oleína y aceite de palma en sus formas naturales o mezclas con otros aceites, se utilizan anualmente para frituras domésticas e industriales; adicionalmente, numerosos trabajos indican las ventajas del aceite de palma en fritura por su mayor estabilidad oxidativa en comparación con otros aceites (Ismail, 2005, Kalogianni et al., 2011, Mozzon et al., 2013).

Por todo lo anterior, para retrasar el deterioro oxidativo del aceite de fritura se usan los antioxidantes, especialmente los naturales los cuales reducen el proceso de oxidación mejorando la calidad organoléptica del producto final. Los antioxidantes en los alimentos determinan el tiempo de vida útil y reducen las perdidas nutricionales; sin embargo, nunca mejoran ni regeneran la calidad de un producto alimenticio altamente oxidado. Los antioxidantes para ser usados en alimentos deben cumplir algunos requisitos como: ser inodoro, insípido, efectivo a bajas concentraciones, fácil de incorporar, que soporte las condiciones de procesamiento, barato, estable en el producto terminado y que no sea tóxico (Zapata et al., 2013, ZapataLuján et al., 2013).

Por todas estas características los antioxidantes naturales han tomado un gran uso, especialmente los extractos de romero que han mostrado alta resistencia térmica y mejor actividad antioxidante que los sintéticos de mayor uso como BHA, BHT y TBHQ en procesos de frituras de papas en oleínas de palma, conservando mejor la calidad del aceite (Che Man et al., 1999, Ismail 2005). Los compuestos responsables de la actividad antioxidante de los extractos de romero son principalmente diterpenos fenólicos tales como el ácido carnósico, carnosol, rosmanol, epirosmanol, isorosmanol, metil carnosato y otros ácidos fenólicos, como el rosmarínico (Sui et al., 2012, Erkan et al., 2008).

Por lo tanto, la industria de alimentos que trabaja en procesos de fritura necesita disponer de métodos adecuados e insumos para controlar sus procedimientos, que permitan asegurar una calidad sensorial óptima de sus productos finales y una estabilidad oxidativa del aceite sometido a altas temperaturas. Por todo eso, en este estudio se realizó un proceso de fritura de pollo, usando como aceite oleína de palma y como estabilizante del proceso oxidativo del aceite un antioxidante de nombre comercial Noxy-Nat de Tecnas S.A (Medellín, Colombia), reportado como natural y que tiene como ingrediente principal extracto de romero; estos insumos se usaron en un proceso discontinuo de fritura y como objetivo fundamental determinar el efecto directo del antioxidante sobre la estabilidad oxidativa del aceite y su vida útil, haciendo un seguimiento del contenido de los hidroperóxidos y de los compuestos polares totales como indicadores del deterioro oxidativo. Todos los experimentos se realizaron por duplicado. Las regresiones fueron 
calculadas con un nivel de significancia del 95\% ( $p<0.05)$, usando el programa Statgraphics Plus versión 5.0 (Statistical Graphics Corp., Rockville, MD).

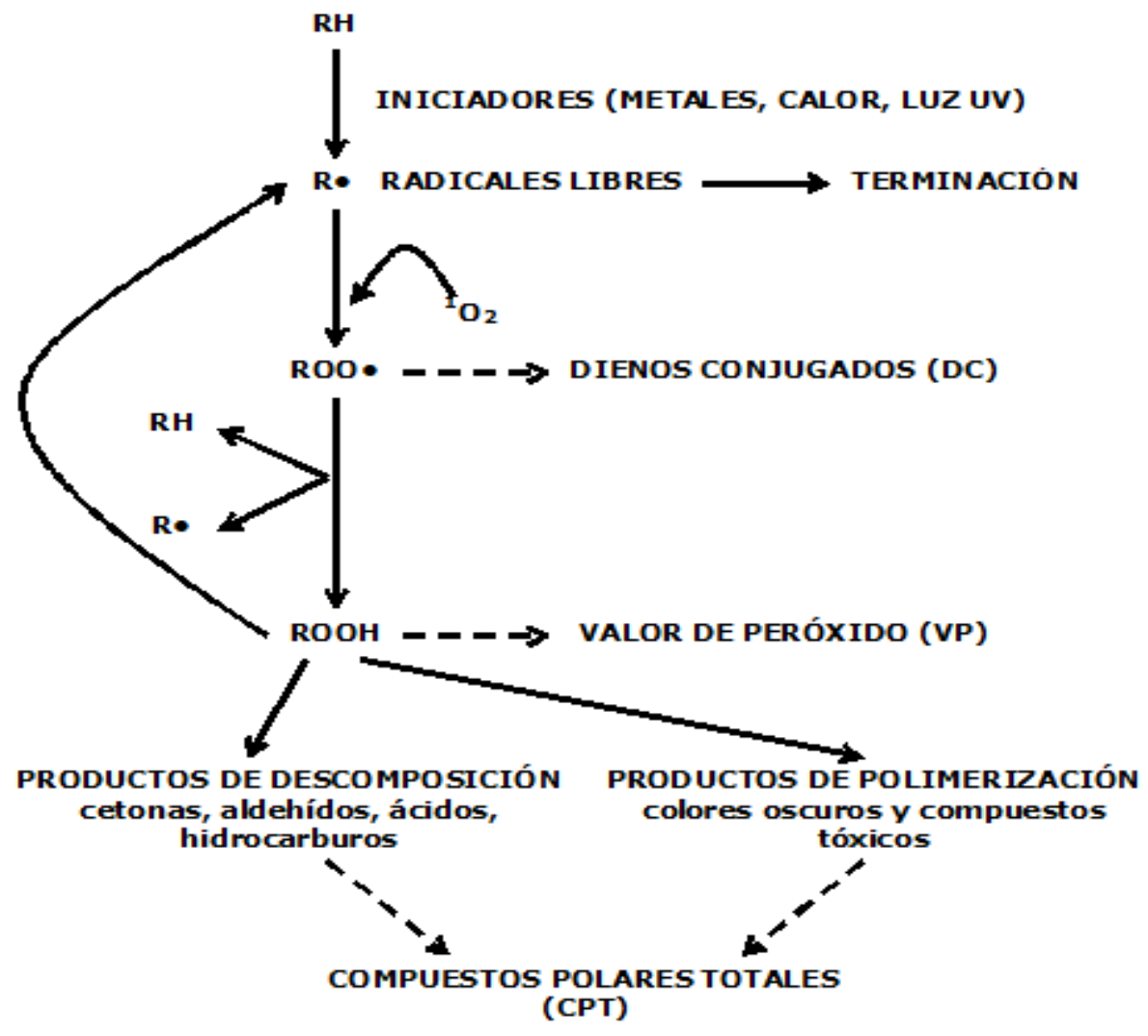

Fig. 1: Oxidación de ácidos grasos insaturados

\section{MATERIALES Y METODOS}

Se realizaron las siguientes técnicas para determinar la actividad antioxidante del Noxy-Nat® de Tecnas S.A (Medellín, Colombia) comparado con un BHT (Butil Hidroxi Tolueno) grado alimenticio suministrado por un proveedor local: Fenoles totales, DPPH (1,1-difenil-2-picrilhidrazilo), FRAP (Ferric Reducing Antioxidant Power), ORAC (Oxygen Radical Absorbance Capacity) hidrofílico y lipofílico. En los ensayos espectrofotométricos se usó un lector de placas Multiskan Spectrum UV-Vis Marca Thermo Scientific, Finlandia, en fluorescencia se usó un espectrofluorímetro Perkin-Elmer LS-55, Beaconstield, U.K. El agua usada en los experimentos es grado HPLC (High Performance Liquid chromatographic), el radical libre 1,1difenil-2-picrilhidrazilo (DPPH), metanol, tricloruro de hierro, 2,4,6 tripiridil triazina (TPTZ), ácido acético, ácido ascórbico, fluoresceína, Trolox, 2,2'-Azinobis (2-amidinopropano) diclorhidrato (AAPH), reactivo de Folin-Ciocalteu, carbonato de sodio, ácido gálico fueron obtenidos de Sigma Aldrich (Colombia).

\section{Fenoles Totales}

La determinación de fenoles se realizó por el método colorimétrico de Folin-Ciocalteu (Singleton et al.,, 1965). En un tubo de reacción se adicionaron $50 \mu \mathrm{L}$ del antioxidante, $425 \mu \mathrm{L}$ de agua destilada y $125 \mu \mathrm{L}$ del reactivo Folin-Ciocalteu. Se agitó y luego se dejó en reposo por 6 minutos. Posteriormente, se adicionaron $400 \mu \mathrm{L}$ de $\mathrm{Na}_{2} \mathrm{CO}_{3}$ al 7,1\%. Después de 1 hora en la oscuridad, se leyó la absorbancia a $760 \mathrm{~nm}$. Se construyó una curva patrón usando como estándar ácido gálico. Los análisis se realizaron por triplicado y los resultados se expresaron como $\mathrm{mg}$ de ácido gálico/L de muestra.

\section{Evaluación de la Capacidad Antioxidante por el método del DPPH}

El ensayo del DPPH expresa la actividad de los compuestos fenólicos solubles en metanol, medio en el cual se conservan sus propiedades moleculares para detener procesos oxidativos iniciados por radicales libres. El 1,1-difenil-1-picrilhidrazilo (DPPH•), es un radical libre estable que presenta una coloración púrpura en medio metanólico, como consecuencia de la donación de un electrón o un protón por un 
compuesto con poder antioxidante la tonalidad desaparece. Este procedimiento se lleva a cabo utilizando 10 $\mu \mathrm{L}$ del compuesto estudiado y $990 \mu \mathrm{L}$ de la solución metanólica de $\mathrm{DPPH}^{\bullet}(20 \mathrm{mg} / \mathrm{L})$. Como referencia se usó la misma cantidad de DPPH y $10 \mu \mathrm{L}$ del solvente de la muestra (DMSO). Luego de 30 minutos de reacción a temperatura ambiente y en la oscuridad, se lee la absorbancia a una longitud de onda de 517 $\mathrm{nm}$. Para cada muestra estudiada se calcula el porcentaje de inhibición del radical y los resultados se expresan como valores TEAC (Trolox Equivalent Antioxidant Capacity) mediante la construcción de una curva patrón usando varias concentraciones de antioxidante TROLOX® (Rojano et al., 2008, Zapata-Zapata et al., 2012).

\section{Ensayo FRAP}

Este método evalúa el poder reductor de una muestra debido a su capacidad para reducir el hierro férrico $\left(\mathrm{Fe}^{+3}\right)$ acomplejado con 2,4,6-tri(2-piridil)-s-triazina (TPTZ) a su forma ferrosa $\left(\mathrm{Fe}^{+2}\right)$, que presenta un máximo de absorbancia a una longitud de onda entre 590-595 nm. $50 \mathrm{uL}$ de muestra, fueron adicionados a 900 uL de una solución de FRAP (Buffer ácido acético-acetato de sodio (pH 3,4), TPTZ, $\mathrm{FeCl}_{3}$, en relación 10:1:1), luego de 30 minutos de reacción se determinó la absorbancia a una longitud de onda de $593 \mathrm{~nm}$, este valor se comparó con la curva de referencia construida con ácido ascórbico como patrón primario, y los resultados fueron expresados como AEAC (Ascorbic Acid Equivalent Antioxidant Capacity: mg de ácido ascórbico /L) (Rojano et al., 2008, Zapata et al., 2013).

\section{Ensayos ORAC Hidrofílico y Lipofílico}

El método consiste en evaluar la degradación oxidativa de la fluoresceína en presencia de iniciadores de radicales libres como los azocompuestos (AAPH), los cuales generan radicales peroxilos (ROO') que interactúan con la fluoresceína. El procedimiento experimental emplea Trolox como estándar y condiciones controladas de temperatura a $37^{\circ} \mathrm{C}$ y pH 7,4 . Las lecturas se realizan a una $\lambda$ de excitación $493 \mathrm{~nm}$ y slit de excitación $10, \lambda$ de emisión $515 \mathrm{~nm}$ y slit de emisión 15 y sin placa atenuadora. Para el desarrollo de la técnica se utilizan soluciones de fluoresceína 1×10-2 M en PBS (75 mM) AAPH 0,6 M en PBS (75 mM). La muestra contiene $21 \mu \mathrm{L}$ de fluoresceína, $2,899 \mu \mathrm{L}$ de PBS, $30 \mu \mathrm{L}$ del extracto ensayado y $50 \mu \mathrm{L}$ de AAPH. Como referencia se usó Trolox. El efecto protector del antioxidante fue calculado usando las diferencias de áreas bajo la curva de decaimiento de la fluoresceína entre el blanco y la muestra, y se comparó contra la curva del Trolox. (Prior et al., 2005, Romero et al., 2010).

\section{Selección de la concentración del Noxy-Nat}

Para determinar la concentración óptima del antioxidante a utilizar en el proceso de fritura se usaron el métodos de oxigeno activo AOM (Active Oxygen Method) y valor de peróxidos (VP) con el aceite sin antioxidante como control, 500 y 1000 ppm de Noxy-Nat y como patrón de comparación BHT a 200 ppm, que es la concentración permitida por el INVIMA (Instituto Nacional de Vigilancia de Medicamentos y Alimentos) de Colombia.

\section{Método AOM}

Una porción de $20 \mathrm{ml}$ de una muestra completamente fundida se coloca en una calentador a una temperatura constante de $99 \pm 0,1^{\circ} \mathrm{C}$ aireada; hasta llegar a un tiempo en el que se alcanza un valor de peróxidos (PV) de 100 meq de $\mathrm{O}_{2} / \mathrm{kg}$ de aceite, bajo la norma $\mathrm{AOM}$ (método de Cd 12-57).

\section{Determinación del valor de peróxidos (VP)}

El valor de peróxidos se determinó por el método de la IDF (International Dairy Federation). Basado en la oxidación de $\mathrm{Fe}^{+2}$ a $\mathrm{Fe}^{+3}$ por los hidroperóxidos y formar un complejo $\mathrm{Fe}^{+3}$-tiocianato determinado por métodos espectrofotométricos.

\section{Proceso de fritura}

\section{Freidora.}

En un proceso discontinuo, se usó una freidora eléctrica de mesa HY 902 marca C.I.TALSA (Medellín, Colombia); un equipo con una dimensión de 58X49X38CM (AXLXH) de dos compartimientos con calderas de aceite: $23 \times 30.5 X 20 \mathrm{CM}$ (AXLXH), para 200V monofásica y un rango de temperaturas entre 25 a $200^{\circ} \mathrm{C}$. 


\section{Índices de calidad del aceite}

A la oleína de palma sin antioxidantes, se le determinaron los índices de calidad y se usó el aceite recién extraído, se midió el valor de acidez según el método oficial AOAC 940.28, el valor de peróxidos (AOAC 965.33) y el índice de yodo (AOAC 993.20).

Los procesos de fritura se desarrollaron usando cortes de pollo en una relación 1:5 en masa respecto al aceite. Un proceso de fritura contiene: $800 \mathrm{~g}$ de pollo y $4.0 \mathrm{~L}$ de aceite calentados a $180^{\circ} \mathrm{C}$, con un tiempo de permanencia de 12 minutos. Realizando 5 frituras diarias y su respectivo muestreo para análisis inmediato y con reposición de aceite al nivel después de cada jornada.

\section{Perfil de ácidos grasos}

El perfil de ácidos grasos del aceite se realizó en un GC 6890N Agilent acoplado a un detector selectivo MS

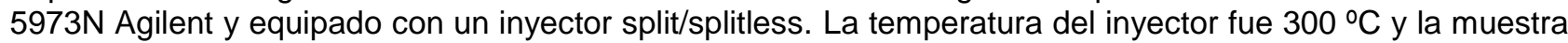
previamente derivatizada con $\mathrm{KOH}$-Metanol fue inyectada automáticamente en el modo Split-less. Se usó una columna HP-5 ms (5\% fenil metil siloxano) de $30 \mathrm{~m}, 0.25 \mathrm{~mm}$ con un espesor de película $0.25 \mu \mathrm{m}$ y una temperatura máxima de $325 \stackrel{\circ}{\circ} \mathrm{C}$. El programa de temperatura se inició a $50{ }^{\circ} \mathrm{C}$ subiendo a $200 \stackrel{\circ}{\circ} \mathrm{C}(5$ $\min )$ y alcanzando una temperatura final de $300^{\circ} \mathrm{C}(14 \mathrm{~min})$ a una rata de $10 \stackrel{\circ}{\circ} / \mathrm{min}$. Se usó helio como gas portador a un flujo constante de $1 \mathrm{~mL} / \mathrm{min}$. La temperatura del detector fue de $300 \stackrel{\circ}{\circ} \mathrm{C}$. El software usado para el cálculo de todos los parámetros fue MSD ChemStation D 02.00.275 Copyright@ Agilent Technologies 1989-2005. Para la determinación de ácidos grasos saturados e insaturados como metilésteres se usó la base de datos NIST 2005 (Zapata-Luján et al., 2013).

\section{Estabilidad oxidativa de la oleína de palma}

Para estimar la estabilidad oxidativa del aceite durante el proceso de fritura, se usó el valor de peróxidos y la determinación de Compuestos Polares Totales (CPT) norma establecida para muchos países Europeos. En nuestro caso consideramos un $27 \%$ de CPT como indicador final del deterioro oxidativo del aceite de fritura.

\section{Determinación de Compuestos Polares Totales (CPT)}

Se determinó el contenido de compuestos polares totales de acuerdo al método de la IUPAC (International Union of Pure and Applied Chemistry) bajo la norma 2.507 de 1987; usando cromatografía de adsorción en columna de sílica gel como fase fija y una mezcla de solventes (hexano: éter etílico (90:10)v/v) como fase móvil, para la elución de los compuestos no polares (los triglicéridos no alterados), y así obtener luego por diferencia de pesos el contenido de compuestos polares y sus respectivos porcentajes (Juárez et al., 2005).

\section{RESULTADOS Y DISCUSIÓN}

\section{Aceite}

Se determinaron los índices de calidad con los siguientes resultados: acidez libre (\% de ácido oleico) $=$ $0.036 \pm 0.005$, valor de peróxido $=0.22 \pm 0.03$, índice de yodo $=60.4 \pm 0.7$. El aceite en la fritura actúa como medio transmisor de calor y llega a ser un ingrediente del producto frito al ser absorbido por el mismo. Esta última función tiene especial interés ya que la estabilidad del aceite y su grado de alteración influirán directamente en la duración del producto frito.

\section{Perfil de ácidos grasos}

En el aceite se detectaron 16 ácidos grasos mayoritarios, con una relación de \% de insaturados/saturados $(\mathrm{l} / \mathrm{S}=1.6)$ y con cadenas largas de 12 a 18 carbonos. En la Tabla 1 se resume el porcentaje de ácidos grasos de la oleína de palma, donde se resaltan el alto contenido de ácido oleico (w9) (31.9), vaccénico (20), 15-tetracosenoico (7.22), esteárico (7.6) y docosanoico (8.43) como los mayoritarios y la baja cantidad (menor del 1\%) de ácidos grasos poliinsaturados. La composición de la oleína de palma refleja la estabilidad frente a los procesos oxidativos como la fritura y mayor vida útil comparados con otros aceites (Mozzon et al., 2013), debido al alto contenido de ácidos grasos mono insaturados como el oleico, vaccénico, 11eicosenoico y 15 -tetracosenoico que sumados dan un $64,66 \%$ y el resto corresponde a ácidos saturados compuestos muy estables químicamente. 
Tabla 1: Perfil de ácidos grasos del aceite de la oleína de palma.

\begin{tabular}{|c|c|c|}
\hline Tiempo $(\mathrm{s})$ & Ácido graso & (\%) de ácidos grasos \\
\hline 19.4 & Palmítico & 5.91 \\
\hline 21.2 & Oleico & 5.51 \\
\hline 22.5 & Esteárico & 31.94 \\
\hline 24.9 & Vaccénico & 7.61 \\
\hline 25.3 & Linoleico & 20.00 \\
\hline 25.9 & 16 -metil octadecanoico & 2.40 \\
\hline 26.2 & 10,13 Eicosadienoico & 0.51 \\
\hline 26.9 & 11 eicosenoico & 0.31 \\
\hline 27.1 & Eicosanoico & 5.50 \\
\hline 29.9 & Docosanoico & 1.04 \\
\hline 30.3 & Pentadecanoico & 8.43 \\
\hline 31.0 & $15-$ Tetracosenoico & 0.35 \\
\hline 31.3 & Tetradecanoico & 7.22 \\
\hline 35.9 & Hexacosanoico & 0.25 \\
\hline 36.7 & & 0.52 \\
\hline
\end{tabular}

\section{Antioxidantes y proceso de fritura}

\section{Concentración óptima del antioxidante}

Para el Noxy-Nat los resultados son: Fenoles Totales: $6769.8 \pm 146.2$; DPPH: $97612.6 \pm 5955.5$ ( $\mu$ mol Trolox /L); FRAP: $12890 \pm 86.8 \mathrm{mg}$ ácido ascórbico/L; ORAC Lipofílico: $278061.1 \pm 18092.2 \mu \mathrm{mol}$ Trolox /L muestra; ORAC hidrofílico: $481133.1 \pm 19226.8 \mu \mathrm{mol}$ Trolox /L muestra; para un ORAC Total $=759194.3$ $\mu \mathrm{mol}$ Trolox/L muestra. De otro lado se compararon los resultados con BHT usado en Colombia en la industria alimenticia especialmente en procesos de estabilización de aceites para fritura con los siguientes valores: DPPH: $33986.57 \pm 4425.5 \mu \mathrm{mol}$ Trolox /L; FRAP: $20939.20 \pm 640.9$ (mg ácido ascórbico/L); ORAC Lipofílico: $175295.8 \pm 6876.6 \mu \mathrm{mol}$ Trolox /L muestra; ORAC hidrofílico: $269090.9 \pm 28167 \mu \mathrm{mol}$ Trolox /L muestra; para un ORAC Total $=444386,7 \mu \mathrm{mol}$ Trolox $/ \mathrm{L}$ muestra.

Para determinar la concentración optima del antioxidante se usó el método AOM, con lo cual encontramos los tiempos estimados para alcanzar un valor de peróxido igual a 100 meq de $\mathrm{O}_{2} / \mathrm{Kg}$ de aceite para cada concentración de Noxy-Nat y BHT; y así poder calcular una relación entre el control y cada una de las concentraciones de antioxidante, la relación más alta permite obtener el mejor valor de concentración del Noxy-Nat para usar en el proceso de fritura, ver figura 2. Se usaron para el Noxy-Nat 500 y 1000 ppm, y para el BHT 200 ppm que es la concentración máxima permitida por el INVIMA como las concentraciones de prueba. A partir de regresiones exponenciales altamente significativas estadísticamente, se calcularon los tiempos de deterioro máximo para alcanzar un VP igual a $100 \mathrm{meq} \mathrm{de} \mathrm{O}_{2} / \mathrm{Kg}$ de aceite y son: Control = $87.3 \mathrm{~h}, 1000 \mathrm{ppm}=122.2 \mathrm{~h} ; 500 \mathrm{ppm}=112.0 \mathrm{~h}$ y BHT $200=105.2 \mathrm{~h}$. Las relaciones respecto al control son: Control 1.0; $1000 \mathrm{ppm}=1.4 ; 500 \mathrm{ppm}=1.28$ y $\mathrm{BHT}=1.16$. De tal manera que la concentración seleccionada para la fritura fue $1000 \mathrm{ppm}$.

Los valores del Noxy-Nat como antioxidante son muy superiores a los obtenidos para el BHT, especialmente Ios valores ORAC tanto hidrofílico como lipofílico y los obtenidos para el ensayo con DPPH; en las técnicas ORAC lipofílico y DPPH se usan respectivamente acetona y metanol como solventes, de tal manera que los componentes polifenólicos menos polares del Noxy-Nat se expresan con una alta capacidad para atrapar radicales libres generados en la oxidación de los ácidos grasos insaturados del aceite. De otro lado, de acuerdo a las metodologías ORAC la dosis de 1000 ppm de Noxy-Nat aporta $760 \mu \mathrm{mol}$ Trolox por litro de aceite, que es mayor a la aportada por el BHT (alrededor de $120 \mu \mathrm{mol}$ Trolox por litro de aceite. Por esto y por efectos de estabilidad de los componentes del romero hay una mayor actividad del Noxy-Nat respecto al BHT para extender el proceso de conservación de la oleína de palma sometida a altas temperaturas. Debido a los altos valores ORAC del Noxy-Nat se puede suponer que los compuestos polifenólicos del Noxy-Nat $(\mathrm{ArOH})$ tienen capacidad para atrapar radicales peróxilos (ROO•) generados en el proceso oxidativo mediante un mecanismo de transferencia de protones (Hydrogen Atom transfer (HAT)), proceso medido en las técnicas ORAC (Che Man et al., 1999, Duque et al., 2013) y estabilizar el proceso (ecuación 1). 


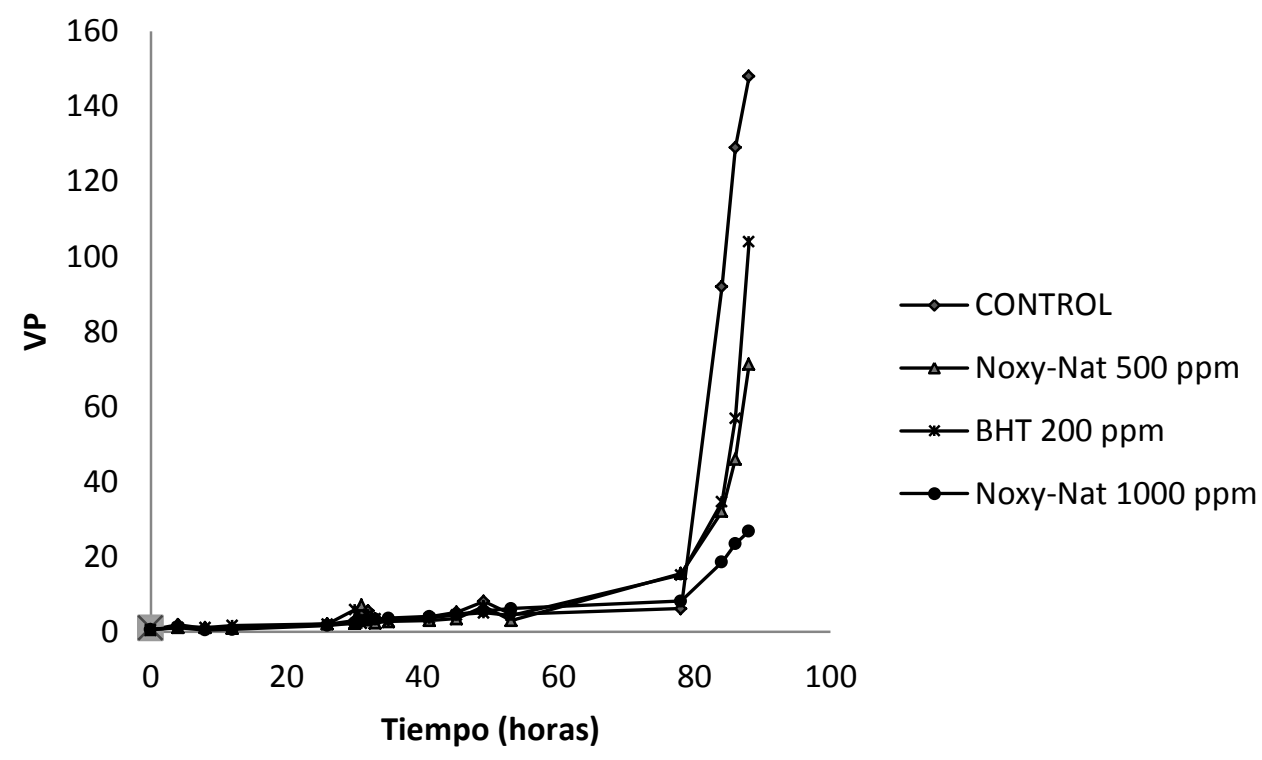

Fig 2. Valor de peróxidos (VP) (meq de $\mathrm{O}_{2} / \mathrm{Kg}$ de aceite) contra el tiempo (h), para seleccionar la concentración óptima del Noxy-Nat.

$\mathrm{ArOH}+\mathrm{ROO} \bullet \rightarrow \mathrm{ArO} \bullet+\mathrm{RH}$

Fritura

En la figura 3, se observa que el valor de peróxidos para el control y el Noxy-Nat a 1000 ppm aumentó a partir del primer día del proceso de fritura, sin embargo se han encontrado en algunos casos disminución de los mismos, porque en el proceso oxidativo los hidroperóxidos se descomponen para generar sustancias más polares altamente oxidadas como aldehídos cetonas y ácidos. Se considera que el aumento de los hidroperóxidos se puede deber a que el aceite en los días de fritura después del calentamiento es enfriado por largos periodos de tiempo (12 horas), y en este tiempo el oxígeno del aire en contacto con los ácidos grasos libres pueden generar nuevos hidroperóxidos (Choe et al., 2006, Tsaknis et al., 2002). En nuestro estudio el control sin antioxidante se disparó exponencialmente a partir del día 5 de fritura mientras que el en el aceite con Noxy-Nat 1000 ppm a partir del día 5 los hidroperóxidos entran en una fase estacionaria. El valor de peróxido por los diferentes comportamientos que presentan en los procesos de fritura no se considera un buen indicador del deterioro oxidativo de aceites de frituras. Sin embargo, en este trabajo se encuentra que el Noxy-Nat 1000 ppm rico en romero, inhibe significativamente el proceso oxidativo de la oleína de palma, cuando se usan los hidroperóxidos como indicadores de deterioro.

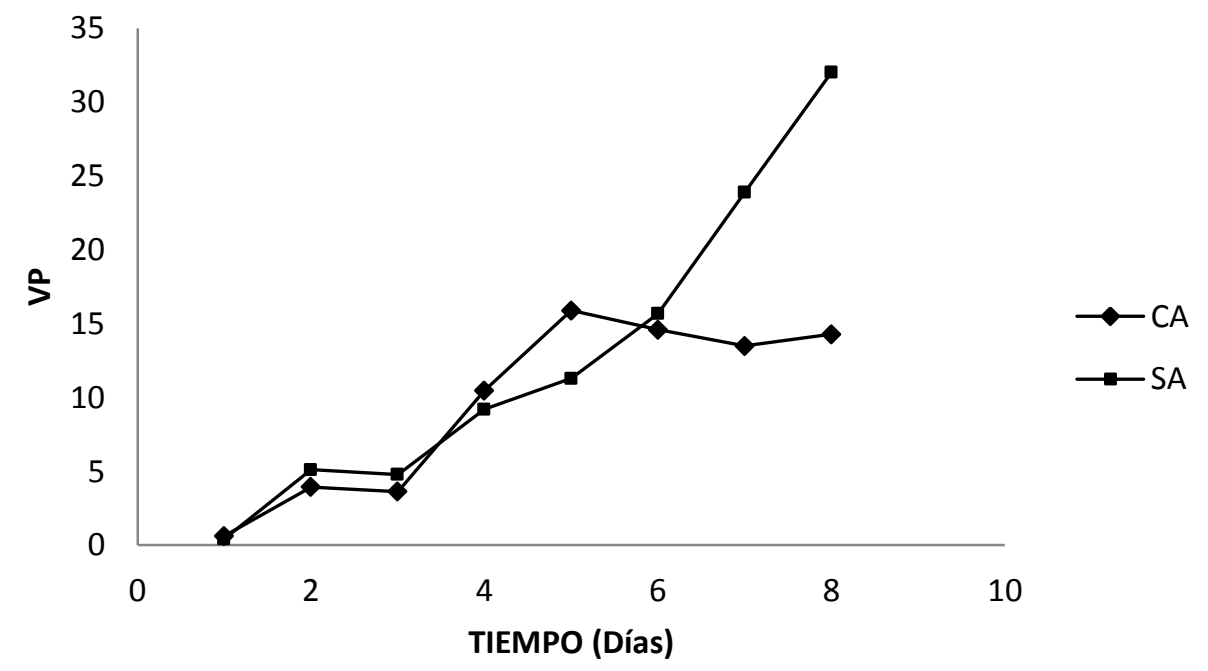

Fig 3. Perfil de valor de peróxidos durante el proceso de Fritura. $S A=$ aceite $\sin$ antioxidante, $C A=$ aceite con 1000ppm de Noxy-Nat 
De otro lado, los compuestos polares son productos finales de la peroxidación y aportan información de nuevos compuestos formados durante el proceso oxidativo y es una de las técnicas más recomendadas para hacer el seguimiento de grasas y aceites en el proceso de fritura (Casal et al., 2010, Chen et al., 2013). La formación de los compuestos polares totales (CPT) está fuertemente relacionada con la oxidación primaria y secundaria que tiene lugar durante el proceso de fritura, y es un índice de calidad establecido para aceites de fritura con límites de rechazo entre $20-27 \%$ debido a los efectos negativos sobre la calidad sensorial (olor, color y sabor) y del valor nutricional del alimento frito; los productos oxidados formados también están relacionados con efectos perjudiciales para la salud humana (Karakaya et al., 2011, Pedreschi et al., 2007).

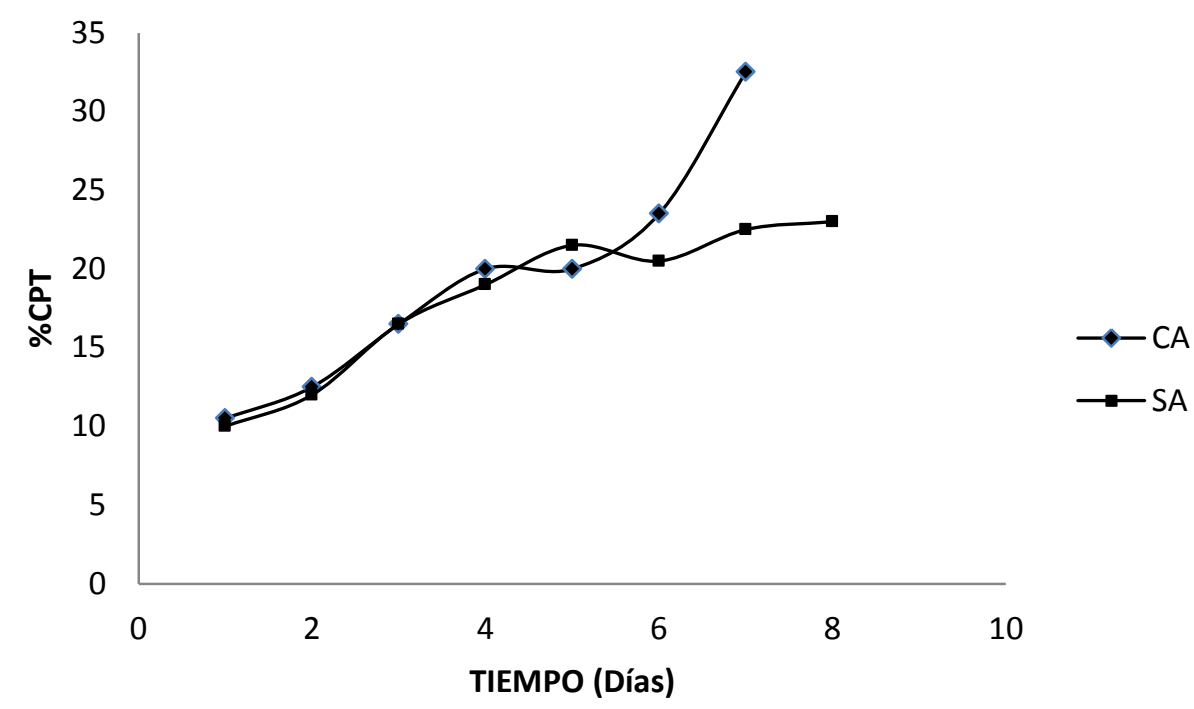

Fig 4. Perfil de los \%CPT en el proceso de fritura. $\mathrm{SA}=$ aceite sin antioxidante, $\mathrm{CA}=$ aceite con 1000ppm de Noxy-Nat.

En Colombia no hay legislación respecto a los límites de calidad de los aceites de fritura, y se usan las pruebas de color como índice de deterioro pese a que el color no es un indicador adecuado del daño oxidativo del aceite en la fritura, sobre todo cuando se usan productos cárnicos o alimentos ricos en metabolitos secundarios coloreados como los carotenoides y algunos flavonoides. Para este trabajo tomamos $27 \%$ de CPT como valor superior para rechazar el aceite usado en la fritura como indicador de deterioro oxidativo. En la figura 4, se observa que a partir del día 5 los compuestos polares totales en el aceite $\sin$ antioxidante se aumentan exponencialmente mientras que en el aceite con Noxy-Nat 1000 ppm el aumento durante todo el proceso es lineal. Usando una regresión exponencial para el aceite sin antioxidante con un $\mathrm{R}^{2}=96.2 \%$ y con la expresión CPT $=\exp (2.1561+0.19078$ días $)$ se obtuvo un tiempo igual a 5.8 días para alcanzar CPT 27\%. Para el aceite con Noxy-Nat 1000 ppm, se obtuvo la expresión lineal PT $=9.66071+1.88095$ días con un $\mathrm{R}^{2}$ del 89, para obtener CPT igual a 27 y una vida útil igual a 9.22 días. De tal manera que el antioxidante Noxy-Nat puede extender el tiempo de vida útil del aceite de palma en un $59 \%$.

\section{CONCLUSIONES}

EL antioxidante natural Noxy-Nat, posee una actividad antioxidante muy alta comparada con el BHT, especialmente la expresada como valores ORAC lipofílico e hidrofilicos y valores TEAC por la metodología DPPH. Con estas propiedades el Noxy-Nat puede ser usado como inhibidor de los procesos oxidativos de grasas y aceites como reemplazante de muchos antioxidantes sintéticos usados en la industria de los alimentos, especialmente en el aumento de la vida útil de aceites puros y en sistemas emulsificados como mayonesas y otros productos lácteos. En este estudio los valores de peróxido permitieron hacer un seguimiento del proceso de fritura; sin embargo no hay una norma con un valor determinado de VP que determine el rechazo del aceite. En cambio, los compuestos polares totales (CPT) son incrementados durante todo el proceso de fritura, de tal manera que durante el proceso oxidativo se pueden expresar los productos iniciales de la peroxidación como los dienos conjugados, los productos intermedios como hidroperóxidos y los productos finales del deterioro oxidativo como algunos compuestos carbonilicos y compuestos polímeros altamente oxidados, por lo tanto la medición de los CPT describe completamente el proceso de oxidación de aceites usados en la industria de la fritura. El Noxy-Nat como antioxidante a 1000ppm retarda 
la oxidación del aceite de palma en un $59 \%$ durante un proceso de fritura de pollo discontinuo realizado a $180^{\circ} \mathrm{C}$, durante $12 \mathrm{~min}$, en una relación Aceite/pollo igual a 5, con 5 frituras diarias y con recambio de aceite a nivel cada día.

\section{REFERENCIAS}

Aladedunye, F y Przybylski, R., Frying stability of high oleic sunflower oils as affected by composition of tocopherol isomers and linoleic acid content. Food Chemistry, 141(3): 2373-2378 (2013)

Alvis, A., Villada, $\mathrm{H}$ y Villada, D., Efecto de la Temperatura y Tiempo de Fritura sobre las Características Sensoriales del Ñame (Dioscorea alata). Información Tecnológica, 19(5): 19-26 (2008).

Alvis A, Vélez C.A., Modelado del proceso de fritura del ñame (Dioscorea alata) mediante mediciones reológicas usando la metodología de superficie de respuesta. Información Tecnológica, 19(5): 11-18 (2008).

Bou, R., y otros 4 autores., Quality assessment of frying fats and fried snacks during continuous deep-fat frying at different large-scale producers. Food Control, 27(1): 254-267 (2012).

Cassal, S., y otros 4 autores., Olive oil stability under deep-frying conditions. Food and Chemical Toxicology, 48(10): 2972-2979 (2010).

Chen, W., y otros 4 autores., Total Polar Compounds and Acid Values of Repeatedly Used Frying Oils Measured by Standard and Rapid Methods. Journal of Food and Drug Analysis, 21(1): 58-65 (2013).

Che Man, Y.B y Tan C.P.T., Effects of Natural and Synthetic Antioxidants on Changes in Refined, Bleached, and Deodorized Palm Olein During Deep-Fat Frying of Potato Chips, J. Am. Oil Chem. Soc. 76(3): 331-340 (1999).

Choe E y Min D.B., Mechanisms and Factors for Edible Oil Oxidation. Comprehensive reviews in food science and food safety, 5(4): 169-186 (2006).

Duque, L., y otros 4 autores, Radical Scavenging Capacity of 2,4-Dihydroxy-9-phenyl-1H-phenalen-1-one: A Functional Group Exclusion Approach. Org. Lett., 15 (14): 3542-3545 (2013).

Erkan, N; Ayranci, G y Ayranci, E., Antioxidant activities of rosemary (Rosmarinus Officinalis L.) extract, blackseed (Nigella sativa L.) essential oil, carnosic acid, rosmarinic acid and sesamol. Food Chemistry, 110(1): 76-82 (2008).

Giua, L., Blasi, F., Simonetti, M.F y Cossignani, L., Oxidative modifications of conjugated and unconjugated linoleic acid during heating. Food Chemistry, 140(4): 680-685 (2013).

International IDF Standards International Dairy Federation, IDF-Square Vergote 41, Brussels, Sec. 74A (1991).

Ismail, R., Palm oil and palm olein frying applications Asia Pac J Clin Nutr, 14 (4): 414-419 (2005).

Juárez, M., Masson, L y Sammán, N., Deterioro del aceite de Soya hidrogenado empleado en la fritura de un alimento cárnico. Grasas y Aceites, 56(1): 53-58, (2005).

Karakaya, S., y Simsek S., Changes in Total Polar Compounds, Peroxide Value, Total Phenols and Antioxidant Activity of Various Oils Used in Deep Fat Frying. J Am Oil Chem Soc, 88:1361-1366, (2011).

Kalogianni, E.P; Karapantsios, T.D y Miller R., Effect of repeated frying on the viscosity, density and dynamic interfacial tension of palm and olive oil. Journal of Food Engineering 105(1): 169-179, (2011)

Mozzon, M., y otros 4 autores., Crude palm oil from interspecific hybrid Elaeis oleifera $\times$ Elaeis guineensis: Fatty acid regiodistribution and molecular species of glycerides. Food Chemistry, 141 (1): 245-252 (2013). 
Official Methods and Recommended Practices of the American Oil Chemists Society, 4th edn., American Oil Chemists' Society, Champaign, (1989).

Pedreschi, F., Kaack, K., Granby, K y Troncoso E., Acrylamide reduction under different pre-treatments in French fries. Journal of Food Engineering, 79(4): 1287-1294 (2007).

Prior, R.I., Wu. X y Schaich, K., Standardized methods for the determination of antioxidant capacity and phenolics in foods and dietary supplements. J. Agric. Food Chem, 3(10): 4290-4302 (2005).

Rojano, B., y otros 6 autores, Experimental and theoretical determination of the antioxidant properties of isoespintanol (2-Isopropyl-3,6-dimethoxy-5-methylphenol). J Mol Struct, 877(1-3): 1-6 (2008).

Romero, M., y otros 5 autores, Antioxidant capacity of pure compounds and complex mixtures evaluated by the ORAC-Pyrogallol Red Assay in the Presence of Triton X-100 Micelles. Molecules. 15(9): 6152-67 (2010).

Singleton, V.L y Rossi, J.A., Colorimetry of totalphenolics with phosphomolybdic-phosphotungstic acid reagents. American Journal of Enology and Viticulture, 16: 144-158 (1965).

Sui, X., y otros 6 autores. Microwave irradiation to pretreat rosemary (Rosmarinus officinalis L.) for maintaining antioxidant content during storage and to extract essential oil simultaneously. Food Chemistry, 131(4): 1399-1405 (2012).

Tsaknis J, Lalas S., Stability during frying of Moringa oleifera Seed Oil Variety "Periyakulam 1". Journal of Food Composition and Analysis, 15(1): 79-101 (2002).

Wu, H., Karayiannis, T.G y Tassou, S.A., A two-dimensional frying model for the investigation and optimisation of continuous industrial frying systems. Applied Thermal Engineering, 51(1-2): 926-936 (2013).

Zapata-Zapata, A.D., y otros 4 autores, Enzymatic maceration of albedo layer from sour orange (Citrus aurantium L.) with protopectinase-se and measurement of antioxidant activity of the obtained products. LWT - Food Science and Technology, 45(2): 289-294 (2012).

Zapata-Luján, A., Cogollo, A y Rojano B., Potencial nutracéutico del aceite de la almendra de choibá o almendro de montaña (Dipteryx oleifera Benth.). Revista Cubana de Plantas Medicinales, 18(3): 1-10 (2013).

Zapata, K., Cortes, F y Rojano, B., Polifenoles y actividad antioxidante del fruto de Guayaba Agria (Psidium araca). Información Tecnológica: En prensa, (2013). 